Research Article,

\title{
Efficacy and Safety for the Combination of Neomycin, Beclomethasone, Clotrimazole and Lignocaine for the Treatment of Otitis Media with Perforation and Otitis Externa: Post-Marketing Surveillance Study
}

\author{
Dr. Mayuresh Kiran ${ }^{1}$, Mr. Lalit Pawaskar ${ }^{2}$, Ms. Pramita Waghambare ${ }^{3}$, Ms. Shaheen Sheikh \\ ${ }^{1}$ Vice president, Medical Services and Pharmacovigilance, Centaur Pharmaceuticals Pvt. Ltd. \\ ${ }^{2}$ Executive, Pharmacovigilance, Centaur Pharmaceuticals Pvt. Ltd. \\ ${ }^{3,4}$ Research associate, Pharmacovigilance, Centaur Pharmaceuticals Pvt. Ltd. \\ Email Address: lalit@ centaurlab.com
}

\begin{abstract}
:
Introduction: Bacterial and/ or fungal infection of the middle or external ear can be painful and sometimes the infection can be of mixed nature i.e. caused due to both bacteria and fungi. The combination of antibiotics and antifungals can be used for the treatment of mixed ear infections, along with it, antiinflammatory agent and anaesthetics can be combined to relieve the discomfort caused by the infection. This study was conducted to test the efficacy and safety for the combination of Neomycin, Beclomethasone, Clotrimazole and Lignocaine (investigational product) for the treatment of middle or external ear infection.

Method: Of enrolled 200, 177 patients completed the study. Efficacy was evaluated by symptom score (SS) scale which was an eleven-point scale ranging from 0 to 10 where 0 was no symptom to 10 was the maximum tolerated symptoms. All patients were asked to report SS on SS scale for ear pain, ear discharge and ear itching on day 1, 3 and 5. Safety assessment was made by analysing the adverse events reported by the patient.

Results: SS of ear pain was reduced from 6.158 at baseline visit to 3.050 at day 3 to 0.468 at day 5. SS of ear discharge reduced from 6.175 at baseline to 2.768 at day 3 to 0.491 at day 5. SS of ear itching reduced from 4.694 at baseline to 1.604 at day 3 to 0.299 at day 5 .

Conclusion: The investigational product was found to be efficacious and safe for the treatment of otitis media and externa.
\end{abstract}

Keywords: Neomycin, Clotrimazole, Beclomethasone, Lignocaine, Otitis media and otitis externa

\section{Introduction:}

Ear infection and inflammation is known as otitis is commonly caused by bacteria as well as fungi. Subtypes of ear infection according to their location are otitis externa, otitis media and otitis interna. Otitis externa is also called as external otitis or swimmer's ear is term that broadly defines the infection or inflammation of external ear including auricle and auditory canal of external ear or outer surface of the tympanic membrane. Bacteria including but not limited to Staphylococcus aureus, Plasmodium aeruginosa and anaerobic bacteria are the most common infectious aetiologies, followed by fungi and mycobacteria. ${ }^{(1,2)}$ Otitis media is a category of infective and inflammatory disorders that affects the middle ear. Otitis media is a leading cause of medical visits around the world and its complications are common causes of preventable hearing loss, particularly in developing countries. externa and media are normally caused due to bacteria or fungi but sometimes it is caused due to both bacteria and fungi which is called as mixed ear infections. Mixed ear infections are generally treated with the topical treatment of combination of antibiotic and antifungal agents. The major 
symptoms experienced by the patient of otitis externa and media are inflammation as well as pain in the ear. For symptomatic treatment, to reduce the pain as well as inflammation, topical local anaesthetic agent and anti-inflammatory agents can be used. So for the complete medical management of otitis media and externa the combination of antifungal, antibacterial, local anaesthetic and antiinflammatory agent can be used. ${ }^{(3)}$ So according to the above statement, the combination of Neomycin, Beclomethasone, Clotrimazole and Lignocaine can be used for the medical management of otitis media and externa.

Neomycin is an aminoglycoside antibiotic that works against a variety of aerobic and facultative anaerobic bacteria. ${ }^{(4)}$ Neomycin exerts antibacterial action by inhibition of protein synthesis. Because of the antibiotic action of Neomycin, it can be used for the topical treatment of otitis media or externa. Clotrimazole inhibits ergosterol synthesis, which slows microbial development and thus it is effective against fungal infection. ${ }^{(5)}$ Fungal infections in the ear can be treated with Clotrimazole $1 \%$ solution. Clotrimazole acts by altering the permeability of the fungal cell wall to destroy individual fungal cells. It binds to phospholipids in the cell membrane and prevents the biosynthesis of ergosterol and other sterols, which are essential for cell membrane formation. ${ }^{(6)}$ Beclomethasone dipropionate is a synthetic glucocorticosteroid with a strong anti-inflammatory effect when applied topically. ${ }^{(7)}$ Beclomethasone Dipropionate gives its anti-inflammatory effect by inhibiting both inflammatory cells (e.g., eosinophils, mast cells, lymphocytes, basophils, neutrophils and macrophages) and release of inflammatory mediators (e.g., eicosanoids, histamine, cytokines and leukotrienes). ${ }^{(3)}$ Lignocaine, also known as "Lidocaine," is an amide local anaesthetic agent. Lignocaine is a drug on the World Health Organization's critical drug list that is considered efficacious, safe and costeffective in any health-care system. ${ }^{(8)}$ Lignocaine Hydrochloride acts as a local anaesthetic by stabilizing the neuronal membrane by inhibiting the ionic fluxes needed for the initiation and conduction of impulses. This is especially useful in the extremely painful ear infection / inflammation. ${ }^{(9)}$

So according to the above-mentioned information the fixed dose combination of Neomycin $(0.5 \%$ $\mathrm{w} / \mathrm{v})$, Beclomethasone $(0.025 \%$ w/v $)$, Clotrimazole $(1 \% \mathrm{w} / \mathrm{v})$ and Lignocaine $(2 \% \mathrm{w} / \mathrm{v})$ can be used for the medical management of otitis externa and/ or otitis media. The objective of this post marketing surveillance study was to evaluate the efficacy and safety for the above-mentioned fixed dose combination on Indian patients for the indication of otitis media and otitis externa.

\section{Methodology:}

This post marketing surveillance (PMS) study was conducted at 12 ENT specialty clinical trial sites all over India. The study design was of nonrandomized, non-comparative and user-initiated nature. The study was conducted for the duration of 5 days. Total 200 patients were recruited for the study out of which 177 completed the PMS study. For the PMS study duration of 5 days, patients were asked to visit the clinical trial site at day 3 (visit 2) and day 5 (visit 3) considering the baseline visit as day 1 (visit 1). Visit on day 3 was considered as re-evaluation visit and day 5 was considered as conclusion visit.

\section{Inclusion and Exclusion criteria:}

Patients of both genders (male and female) of age between 18 to 75 years with confirmed diagnosis of otitis media with perforation and externa were recruited for the study. Also, for the study only patients were recruited who were ready to strictly adhere to the study protocol and signed the informed consent form.

Patients with hypersensitivity to Neomycin, Beclomethasone Dipropionate, Clotrimazole or Lignocaine were excluded from the study. Also pregnant and lactating women as well as psychiatric patients were excluded from the study.

\section{Investigational product:}

The investigational product used in the study was the fixed dose combination of Neomycin $(0.5 \%$ $\mathrm{w} / \mathrm{v})$, Beclomethasone Dipropionate $(0.025 \% \mathrm{w} / \mathrm{v})$, Clotrimazole $(1 \% \mathrm{w} / \mathrm{v})$ and Lignocaine Hydrochloride $(2 \% \mathrm{w} / \mathrm{v})$ in the form of ear drops formulation. The investigational product was dispensed to the patient by the investigator at no cost and to the investigator it was supplied at no cost by the sponsor. Patients were asked to instil two drops of investigational product in affected ear 3 times a day. Patients were asked not to take any pharmacological intervention addition to the investigational product in the study duration for the treatment of otitis media or externa.

\section{Study Procedure:}

All eligible patients as per the inclusion and exclusion criteria were enrolled for the PMS study 
by the investigators. The study was conducted for the duration of 5 days to determine the efficacy and safety of the investigational product. All eligible patients were well informed about the PMS study procedure and the investigational product by the investigator and all the doubts were cleared before recruiting to the study. A detailed medical history was obtained from all enrolled patients which was followed thorough the PMS study. Investigational product was dispensed to the patients recruited in the study and were asked to instil two drops to the affected ear 3 times a day for the study duration of 5 days. Patients were instructed to keep a diary of daily symptoms to detect the adverse event if any. For the patients recruited in this study, three visits were planned for the efficacy and safety analysis including baseline visit or visit 1 which was on day 1 , visit 2 (reevaluation visit) on day 3 and visit 3 (conclusion visit) on day 5.

\section{Efficacy Assessment:}

In the PMS study duration of 5 days, the efficacy assessment was done on day 1,3 and 5 by the calculating the decrease in the symptom score (SS) separately for ear pain, ear discharge and ear itching related to otitis externa and/ or otitis media. Symptom score was measured over symptom score scale, which was an eleven-point scale ranging from 0 to 10 where 0 was no symptom to 10 was the highest tolerated symptoms. Patients were asked to rate the symptom score separately for ear pain, ear discharge and ear itching on symptom score scale. The symptom score was further extrapolated to 4 grades as no symptom (0 on SS), mild intensity symptom (1-3 on SS), moderate intensity symptoms (4-6 on SS) and severe intensity symptoms (7-10 on SS) on symptom severity scale.

\section{Safety Assessment:}

Patients were asked for any adverse events on day 3 and 5. These adverse events were categorized into serious and non-serious adverse events and also the causality assessment was done for each reported adverse event.

\section{Regulatory Matters:}

This combination is approved in India for manufacturing and marketing. The informed consent form was read and signed willingly by all of the patients recruited in the study.

\section{Results:}

This PMS study was conducted at 12 clinical trial sites. Total 200 patients were selected for the study out of which 177 completed the study. Mean age of the patients completed the study was 42 years. Out of 177 patients, 84 were male and 93 were female. Efficacy evaluation parameters for the PMS study were symptom score for ear discharge, ear pain and itching which are separately discussed below. Patients had mean symptom score of ear pain 6.158 at baseline, which was reduced to 3.050 at day 3 and was further reduced to 0.468 at day 5 . Graphical presentation for the symptom score of the ear pain at day 1, 3 and 5 is given below in figure 1.

At visit 2 and 3, the reduction in the mean symptom score of ear pain as compared to baseline was found to be $50.458 \%$ and $92.385 \%$ respectively which is graphically presented below in figure 2.

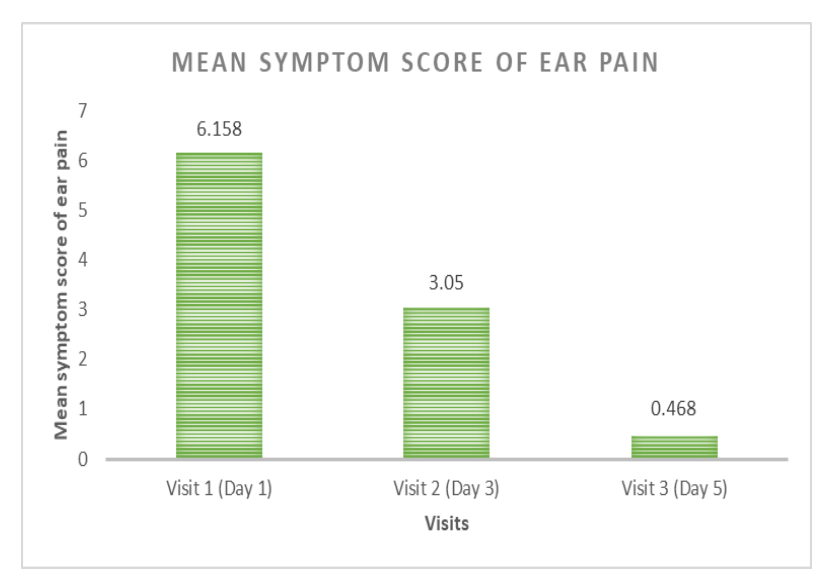

Figure: 1 Mean symptom score of ear pain at day 1, 3 and 5

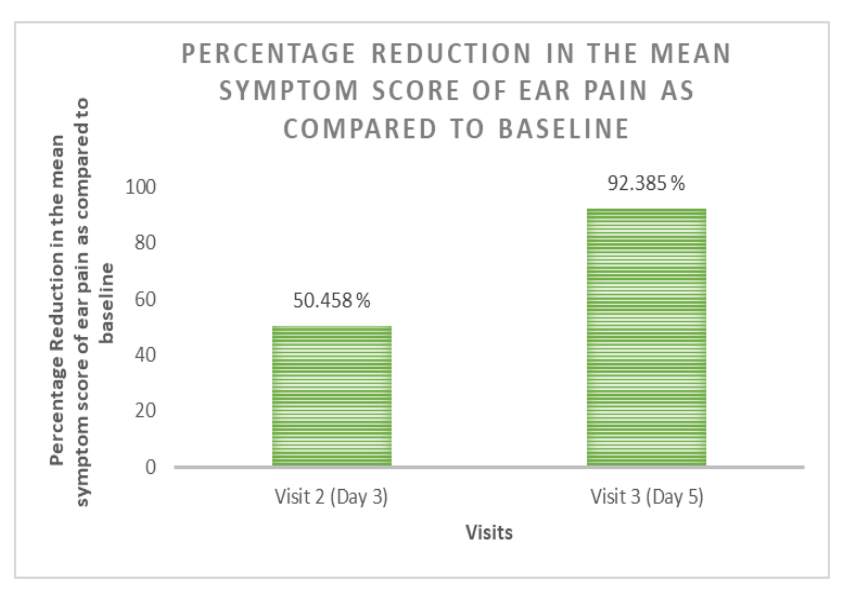

Figure: 2 Percentage reduction in mean symptom score of ear pain at day 3 and 5 as compared to baseline 
Dr. Mayuresh Kiran et al. / Efficacy And Safety For The Combination Of Neomycin, Beclomethasone, Clotrimazole And Lignocaine For The Treatment Of Otitis Media With Perforation And Otitis Externa: Post-Marketing Surveillance Study

The symptom score data of the ear pain was further extrapolated to symptom severity scale. At baseline visit, 51 (28.81\%) patients had severe intensity symptoms, 113 (63.84\%) had moderate intensity symptoms and 13 (7.34\%) had mild intensity symptoms of ear pain. At day 3, only 3 (1.69\%) patients had severe intensity symptoms, 64 (36.15\%) had moderate intensity symptoms and $89(50.28 \%)$ had mild intensity symptoms, whereas 21 (11.86 \%) patients had no symptom of ear pain. At day 5 , $105(59.32 \%)$ patients had no symptom, 67 (37.85\%) patients had mild intensity symptoms and only 5 (2.82\%) had moderate intensity symptoms and no patient had severe intensity symptom of ear pain.

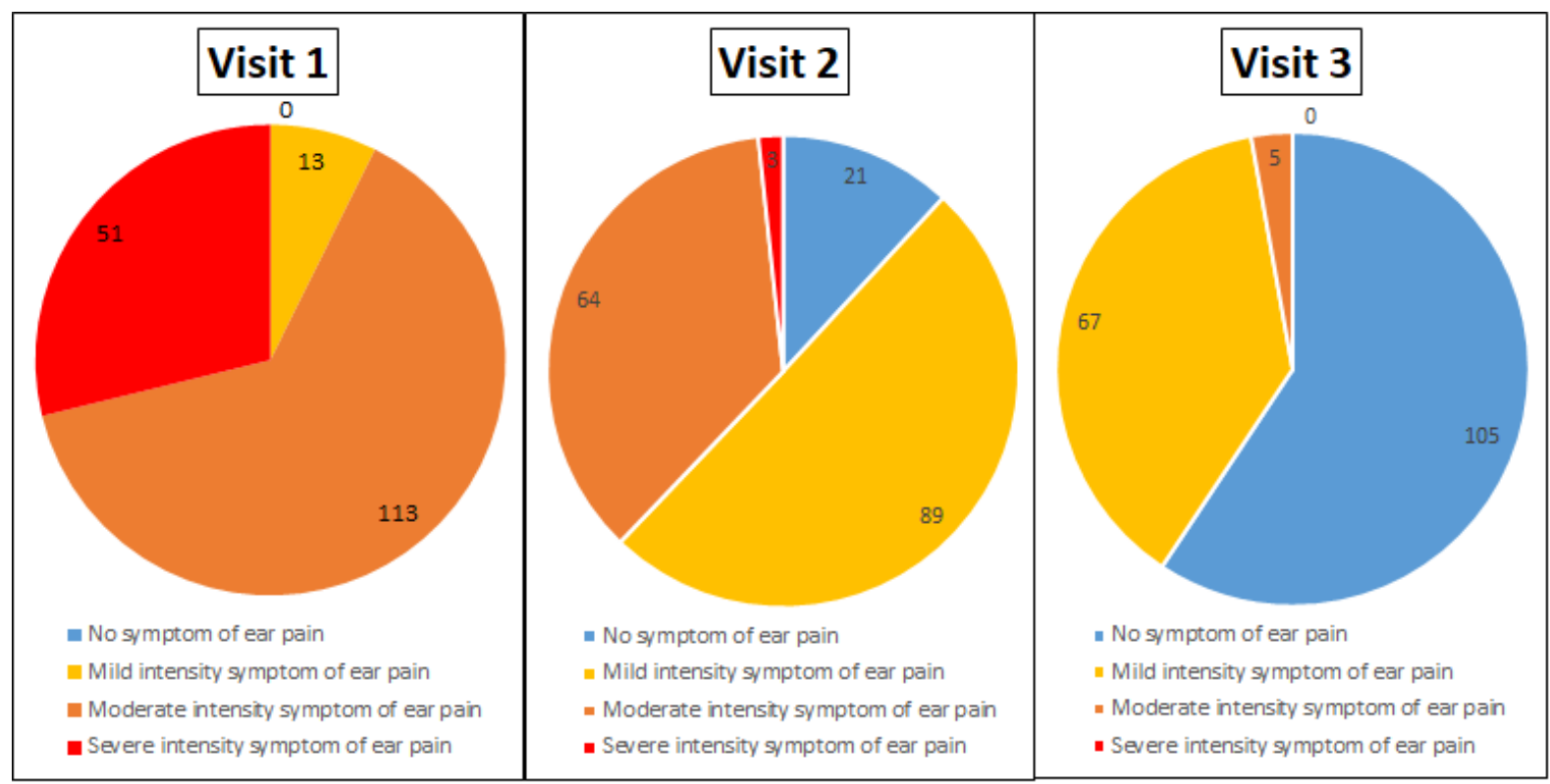

Figure: 3 No of patients with mild, moderate and severe intensity symptoms of ear pain or of no ear pain at Visit 1, 2 and 3

Mean symptom score of ear discharge at baseline visit was 6.175, which was reduced to 2.768 at day 3 and was further reduced to 0.491 on day 5, which is graphically presented below in figure 4.

On day 3 and day 5, the percentage reduction in the mean symptom score of ear discharge was $55.169 \%$ and $92.040 \%$ as compared to the baseline which is graphically presented below in figure 5.

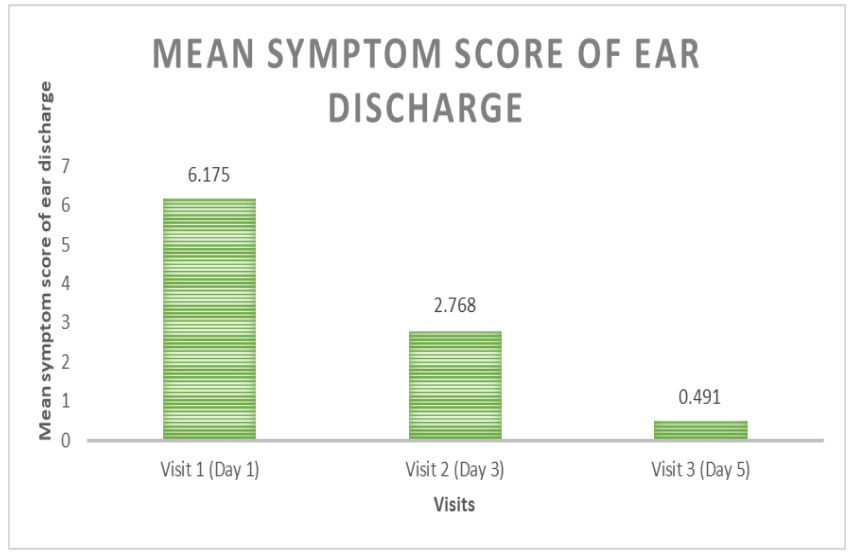

Figure.4: Mean symptom score of ear discharge at day 1, 3 and 5

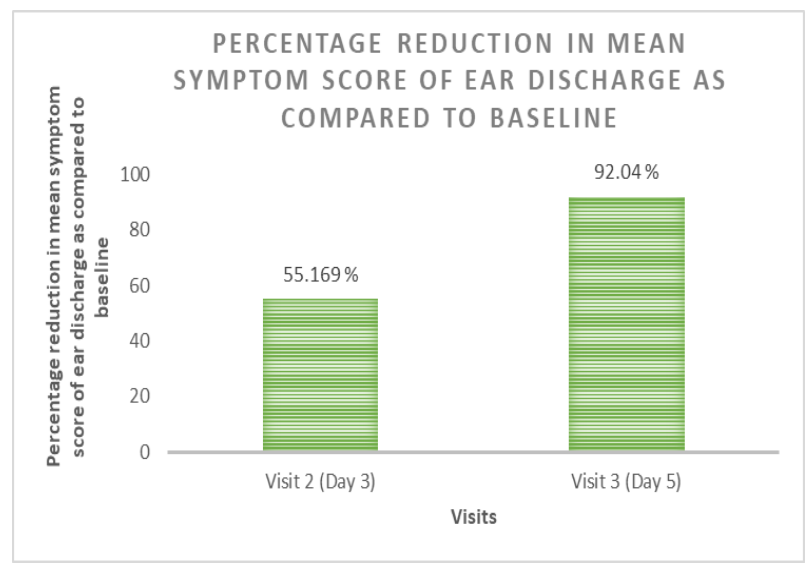

Figure 5: Percentage reduction in mean symptom score of ear discharge at day 3 and 5 as compared to baseline. 
Dr. Mayuresh Kiran et al. / Efficacy And Safety For The Combination Of Neomycin, Beclomethasone, Clotrimazole And Lignocaine For The Treatment Of Otitis Media With Perforation And Otitis Externa: Post-Marketing Surveillance Study

The symptom score data of the ear discharge was further extrapolated to symptom severity scale. At baseline visit, 42 (23.72 \%) patients had severe intensity symptoms, 118 (66.66\%) had moderate intensity symptoms and $17(9.60 \%)$ had mild intensity symptoms of ear discharge. At day 3, only 2 (1.12\%) patients had severe intensity symptoms, 57 (32.20\%) had moderate intensity symptoms and 90 (50.84\%) had mild intensity symptoms, whereas 28 (15.81\%) patients had no symptom of ear discharge. At day 5, 101 (57.06 \%) patients had no symptom, 73 (41.24\%) patients had mild intensity symptoms and only 3 (1.69\%) had moderate intensity symptoms and no patient had severe intensity symptom of ear discharge.

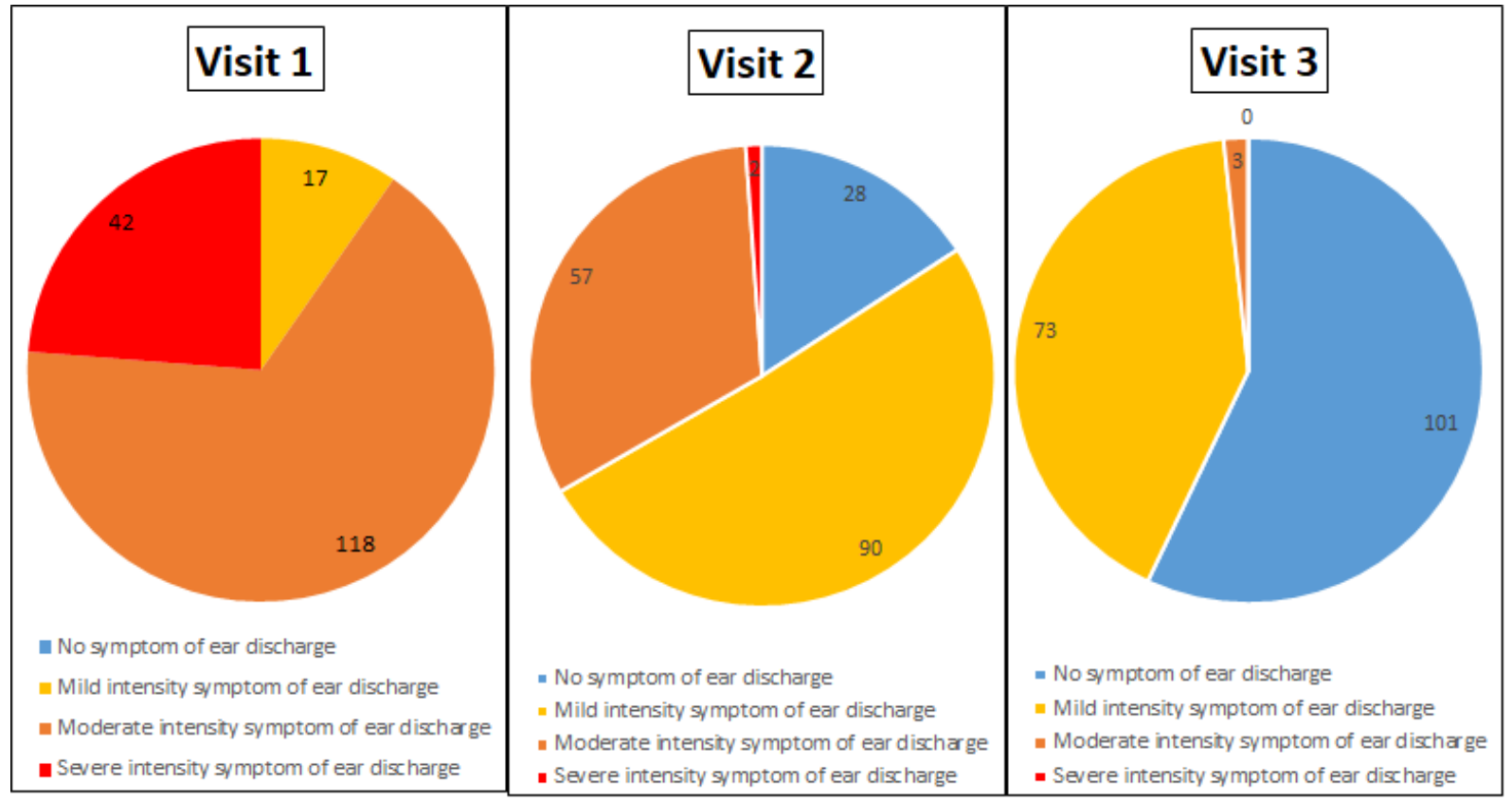

Figure6: No. of patients with mild, moderate and severe intensity symptoms of ear discharge or of no ear discharge at visit 1, 2 and 3

Mean symptom score of ear itching at baseline was 4.694 which was reduced to 1.604 at day 3 and was further reduced to 0.299 at day 5 which is graphically presented below in figure 7.

At day 3 and 5, the percent reduction in the symptom score of ear itching was $65.824 \%$ and $93.622 \%$ as compared to the baseline which is graphically presented below in figure 8.

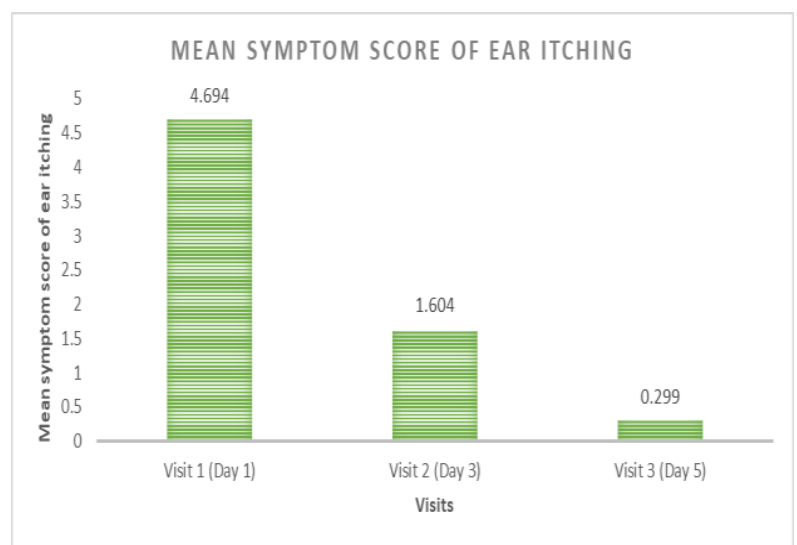

Figure: 7 Mean symptom score of ear itching at day 1, 3 and 5

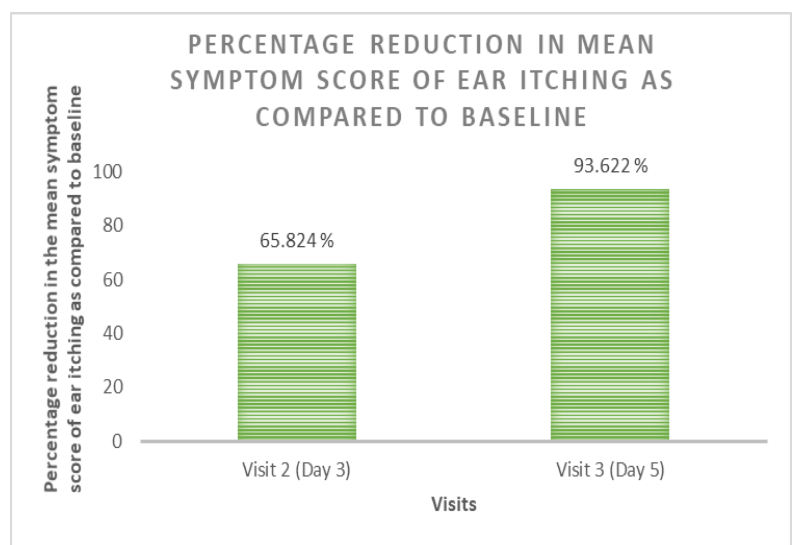

Figure: 8 Percentge Reduction in mean symptom score of ear itching as compared to baseline 
The symptom score data of the ear discharge was further extrapolated to symptom severity scale. At baseline visit, 58 (32.76 \%) patients had severe intensity symptoms, 101 (57.06\%) had moderate intensity symptoms and 18 (10.16\%) had mild intensity symptoms. At day 3, no patient had severe intensity symptoms, 62 (35.02\%) had moderate intensity symptoms and 105 (59.32 \%)

had mild intensity symptoms, whereas 10 (5.64\%) patients had no symptom of ear discharge. At day 5, 112 (63.27\%) patients had no symptom, $64(36.15 \%)$ patients had mild intensity symptoms and only 1 (0.56\%) had moderate intensity symptoms and no patient had severe intensity symptom.

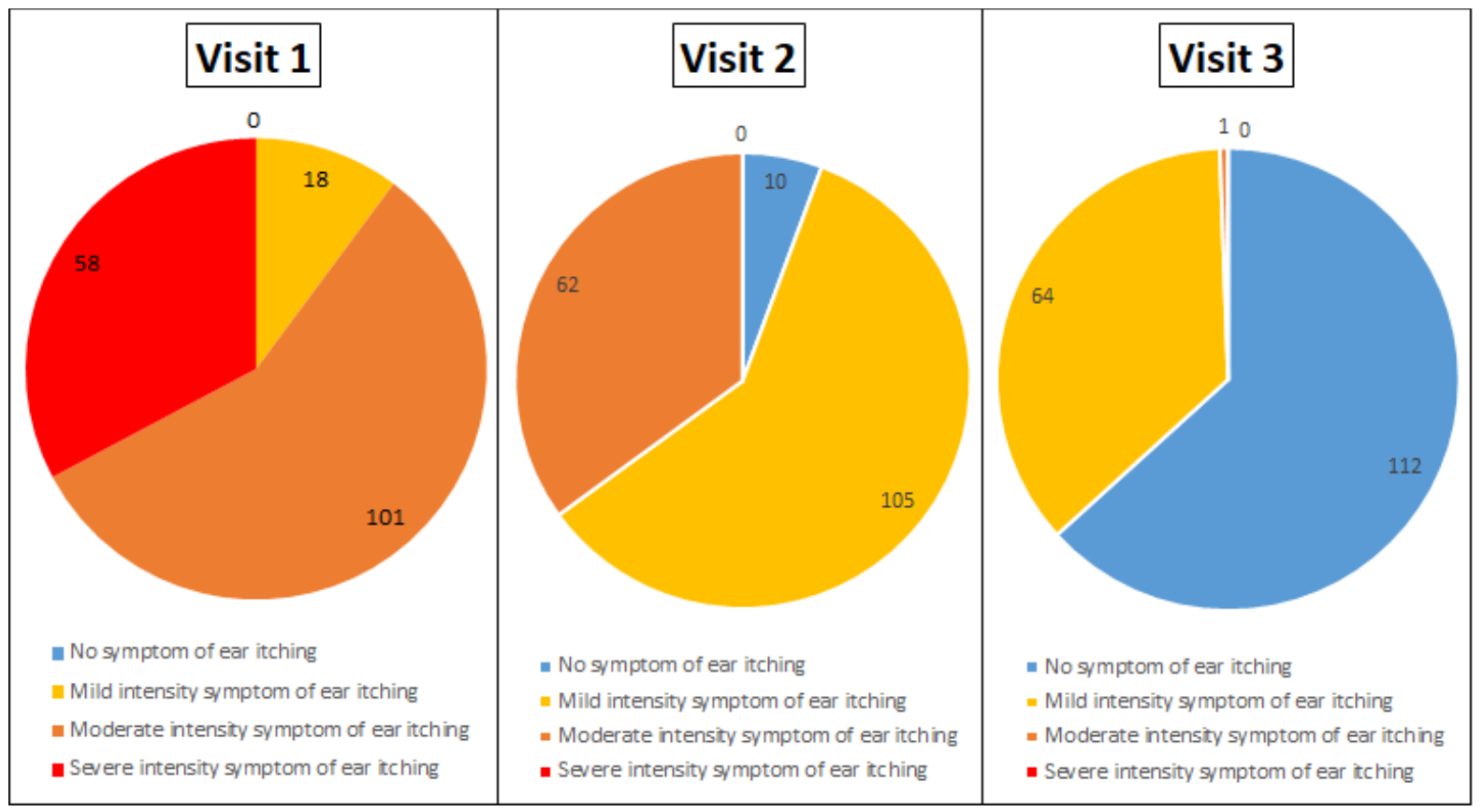

Figure.9: No. of patients with mild, moderate and severe intensity symptoms of ear itching or of no ear itching at visit 1, 2 and 3

\section{Safety Assessment:}

During the study, 9 adverse drug reactions (ADR) of burning sensation of the ear were reported at the site of application. The ADR reported was of nonserious in nature and mild in intensity.

\section{Discussion:}

Otitis media and externa are the most common problems for both children and adults, but the severity varies by the geographical location, with developed countries having the highest prevalence. It is estimated to be the most common disease for which patients visit physicians. ${ }^{(10)}$ Mixed ear infections can be treated by the topical treatment by the combination of antibiotic and antifungal agents. The major symptoms experienced by the patient of otitis externa and media are inflammation as well as pain in the ear. For symptomatic treatment, to reduce the pain as well as inflammation, topical local anaesthetic agent and anti-inflammatory agents can be used. So for the complete medical management of otitis media and externa the combination of antifungal, antibacterial, local anaesthetic and anti- inflammatory agent can be used. ${ }^{(3)}$ So according to the above statement, the combination of Neomycin, Beclomethasone, Clotrimazole and Lignocaine can be used for the medical management of otitis media and externa. This study was conducted to test the efficacy and safety for the treatment of otitis media and externa for the fixed dose combination of Neomycin $(0.5 \% \mathrm{w} / \mathrm{v})$, Beclomethasone Dipropionate $(0.025 \% \quad \mathrm{w} / \mathrm{v})$, Clotrimazole $(1 \% \mathrm{w} / \mathrm{v})$ and Lignocaine Hydrochloride $(2 \% \mathrm{w} / \mathrm{v})$ in the form of ear drop formulation. Total 200 patients were enrolled for the study out of which 177 patients completed the study. Efficacy for the investigational product was evaluated by symptom score scale which was an eleven-point scale ranging from 0 to 10 where 0 was no symptom to 10 was the maximum tolerated symptoms. All patients were asked to report symptom score on symptom score scale for ear pain, ear discharge and ear itching on day 1, 3 and 5 which was considered as visit 1, 2 and 3. Safety assessment was made by analysing the adverse events reported by the recruited patient. Symptom score of ear pain was reduced from 6.158 at 

Lignocaine For The Treatment Of Otitis Media With Perforation And Otitis Externa: Post-Marketing Surveillance Study

baseline visit to 3.050 at day 3 to 0.468 at day 5 . At day 3 and 5, the reduction in the symptom score of ear pain was found $50.45 \%$ and $92.38 \%$ respectively as compared to the baseline. Symptom score of ear discharge reduced from 6.175 at baseline to 2.768 at day 3 to 0.491 at day 5 . At day 3 and 5 the reduction in the symptom score of ear discharge was $55.169 \%$ and $92.040 \%$ respectively as compared to baseline. Symptom score of ear itching reduced from 4.694 at baseline to 1.604 at day 3 to 0.299 at day 5 . At day 3 and 5 , the reduction in the symptom score of ear itching was $65.824 \%$ and $93.622 \%$ respectively as compared to the baseline. 9 episodes of adverse drug reactions were reported and all of them were of expected and non-serious nature. All 9 episodes of adverse drug reactions reported were of burning sensation of the ear at the site of application. Below we have also discussed some of the studies which supports the post marketing surveillance study we have conducted.

Abelardo et al. conducted a double blind randomized clinical trial to compare the efficacy of topical steroid (Betamethasone Sodium phosphate) and topical steroid (Betamethasone sodium phosphate) in addition to topical antibiotic (Neomycin Sulphate). Patients recruited to the study were either randomized to Betamethasone Sodium Phosphate $0.1 \%$ or Betamethasone Sodium phosphate $0.1 \%$ with Neomycin Sulphate $0.5 \%$. Study was conducted in United Kingdom for the duration of 2 weeks. For the study, 45 patients were recruited having otitis externa based on the presence of oedema, discharge or debris in the outer ear canal. Patients visual analogue symptom (VAS) scores for pain, blockage, itching and discharge for otitis externa before treating patient and on day 15 were recorded. Both the groups showed statistically similar symptom score at day 0 when no patient was treated with either medication. The change in mean symptom score was $82.8 \%$ and $47.8 \%$ in the group of patients treated with the combination of Betamethasone Sodium phosphate $0.1 \%$ with Neomycin Sulphate $0.5 \%$ and Betamethasone Sodium Phosphate $0.1 \%$ alone respectively. All patients treated with the combination of Beclomethasone and Neomycin showed symptom improvement but in the group of patients treated with Beclomethasone alone, 5 patients got worse. It was concluded by the study that, the combination of topical antibiotic (Neomycin Sulphate) in addition to topical steroid (Betamethasone sodium phosphate) was better as compared to topical steroid (Betamethasone sodium phosphate) alone for the symptomatic control of otitis externa. ${ }^{(11)}$

Kiran et al. conducted the clinical trial to investigate the efficacy and safety for the combination of Ofloxacin $0.3 \% \mathrm{w} / \mathrm{v}$, Clotrimazole $1 \% \mathrm{w} / \mathrm{v}$, Beclomethasone Dipropionate $0.025 \%$ w/v and Lignocaine Hydrochloride $2 \%$ w/v to test its efficacy and safety for the treatment of otitis media with perforation and otitis externa. The study was conducted on 189 patients out of which 174 completed the study. The study was conducted for the duration of 5 days. Efficacy assessment was made by reduction in visual analogue scale (VAS) score of ear itching, ear discharge and ear pain related to otitis media with perforation and otitis externa. Reduction in mean VAS score was observed from 4.12 at baseline to 2.293 at day 3 to 0.54 at day 5 for ear pain, from 4.011 at baseline to 1.931 at day 3 to 0.563 at day 5 for ear discharge and from 2.896 at baseline to 1.31 at day 3 to 0.241 at day 5 for ear itching. Nearly all the patients had $>50 \%$ reduction in their VAS score at all visits in all the parameters except in visit 2 in ear pain where reduction was $44.34 \%$. Complete symptom relief was also observed from the majority of the patients on day 5. It was concluded that the combination of Clotrimazole, Ofloxacin, Lignocaine and Beclomethasone Dipropionate was efficacious and safe for the treatment of otitis media and otitis externa. ${ }^{(3)}$

Kiran et al. conducted the clinical trial to investigate the efficacy and safety for the fixed dose combination of Neomycin, Clotrimazole, Beclomethasone Dipropionate and Lignocaine for the treatment of otitis media and otitis externa of mixed ear infection nature. The study was conducted on 196 patients out of which 160 completed the study. Efficacy assessment was done by analysing the reduction in total symptom score (TSS) of ear discharge, ear itching and ear pain related to otitis media with perforation and Otitis Externa. Reduction in mean TSS was from 5.73 at baseline to 2.19 at day 3 to 0.21 at day 5 for ear pain, from 5.63 at baseline to 1.73 at day 3 to 0.15 at day 5 for ear discharge and from 4.84 at baseline to 1.48 at day 3 to 0.13 at day 5 for ear itching. Also, no serious adverse drug reaction was found. So it was concluded that the combination of Neomycin, Clotrimazole, Beclomethasone Dipropionate and Lignocaine was efficacious and safe for the treatment of otitis media and externa of mixed infection nature. ${ }^{(6)}$ 


\section{Conclusion:}

The fixed dose combination of Neomycin $(0.5 \%$ w/v), Beclomethasone Dipropionate $(0.025 \%$ $\mathrm{w} / \mathrm{v})$, Clotrimazole $(1 \% \mathrm{w} / \mathrm{v})$ and Lignocaine Hydrochloride (2\% w/v) ear drops was efficacious and safe for the treatment of otitis media with perforation and otitis externa of mixed infection nature.

\section{Acknwoledgement:}

We would like to acknowledge Dr. R. Venkata Krishna (Telangana), Gopal Kumar Jha (Bihar), Ehtesham Ahamad Raushan (Bihar), Harish Kumar Nagesh (Telangana), Kasturi Sateesh (Telangana), Raj Kumar Chaoudhary (Bihar), Chinamaya Sundar Ray (Odisha), Sachinadanda Panda (Odisha), Monalisa Patsani (Odisha), Supriya Santosh Khadtare (Maharashtra), Suraj Marutrao Chavan (Maharashtra), Rahul Vijayrao Dake (Maharashtra) who were investigators for this post marketing surveillance study.

\section{Disclosure:}

This post marketing surveillance study was conducted as a part of Pharmacovigilance activity for the fixed dose combination of Neomycin $(0.5 \%$ $\mathrm{w} / \mathrm{v})$, Beclomethasone $(0.025 \% \mathrm{w} / \mathrm{v})$, Clotrimazole $(1 \% \mathrm{w} / \mathrm{v})$ and Lignocaine $(2 \% \mathrm{w} / \mathrm{v})$ which is available in the Indian market under the brand name of Otiflox New Ear Drops manufactured and marketed by Centaur Pharmaceuticals Pvt. Ltd.

\section{References:}

[1] Nnadede PC. Pattern of chronic suppurative otitis media among patients diagnosed with pulmonary tuberculosis seen in federal medical centre, umuahia. Faculty of otorhinolaryngology. 2015.

[2] Roland PS, Stroman DW. Microbiology of acute otitis externa. The Laryngoscope. 2002 Jul;112(7):1166-77.

[3] Kiran M, George S, Pawaskar L. Safety and Efficacy of a topical combination of Ofloxacin, Clotrimazole, Beclomethasone and Lignocaine for management of Mixed Ear Infections: Phase IV Study. International Journal of Medical Science and Innovative Research. 2017;2(3):42-9.

[4] Mingeot-Leclercq MP, Glupczynski Y, Tulkens PM. Aminoglycosides: activity and resistance. Antimicrobial agents and chemotherapy. 1999 Apr 1;43(4):727-37.
[5] Kadavakollu S, Stailey C, Kunapareddy CS, White S. Clotrimazole as a cancer drug: a short review. Medicinal chemistry. 2014;4(11):722.

[6] Kiran M, George S, Pawaskar L. Safety and efficacy of a topical combination of Neomycin, Clotrimazole, Beclomethasone and Lignocaine for management of ear infections: phase IV study. International Journal of Medical Science and Innovative Research. 2017;2(3)50-7.

[7] Libretto SE. Review of the toxicology of beclomethasone dipropionate. Archives of toxicology. 1995 Aug 1;69(8):509-25.

[8] Weinberg L, Peake B, Tan C, Nikfarjam M. Pharmacokinetics and pharmacodynamics of lignocaine: A review. World Journal of Anesthesiology. 2015 Jul 27;4(2):17-29.

[9] Becker DE, Reed KL. Essentials of local anesthetic pharmacology. Anesthesia progress. 2006 Sep 1;53(3):98-109.

[10] Bolt P, Barnett P, Babl FE, Sharwood LN. Topical lignocaine for pain relief in acute otitis media: results of a double-blind placebo-controlled randomised trial. Archives of disease in childhood. 2008 Jan 1;93(1):40-4.

[11] Abelardo E, Pope L, Rajkumar K, Greenwood R, Nunez DA. A double-blind randomised clinical trial of the treatment of otitis externa using topical steroid alone versus topical steroid-antibiotic therapy. European Archives of Oto-RhinoLaryngology. 2009 Jan;266(1):41-5. 